\title{
Survival Prognosis, Tumor Immune Landscape, and Immune Responses of PPP1R18 in Kidney Renal Clear Cell Carcinoma and Its Potentially Double Mechanisms
}

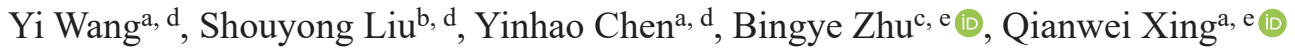

\begin{abstract}
Background: The aim of this study was to investigate the immunological and prognostic roles of protein phosphatase 1 regulatory subunit 18 (PPP1R18) for overall survival (OS) in kidney renal clear cell carcinoma (KIRC) patients, as well as the prediction of its potential mechanisms.
\end{abstract}

Methods: Based on PPP1R18 single gene expression matrices and clinical information from The Cancer Genome Atlas (TCGA), International Cancer Genome Consortium (ICGC) and GSE6344 datasets, the relationships between PPP1R18 and prognosis/immunity were fully explored. Univariate and multivariate Cox regression analyses were applied to assess its independent prognostic ability and gene set enrichment analysis (GSEA) was implemented to find its related pathways. Besides, we also explored possible mechanisms of PPP1R18 involved in KIRC.

Results: PPP1R18 was highly expressed in KIRC samples than in non-tumor tissues in TCGA, ICGC and GSE6344 datasets, with validations from quantitative real-time polymerase chain reaction (qRTPCR) in both cell lines and KIRC tissues (all $\mathrm{P}<0.05$ ). Univariate and multivariate Cox regression analyses indicated that PPP1R18 was also considered to be with independent prognostic ability in KIRC (both $\mathrm{P}<0.01$ ). GSEA results showed that PPP1R18 gene expression was significantly related to Chemokine, JAK STAT, MAPK, and NOTCH pathways. Furthermore, PPP1R18 was also firmly associated with microsatellite instability (MSI), tumor mutational burden

Manuscript submitted January 18, 2022, accepted February 22, 2022

Published online February 28, 2022

aDepartment of Urology, Affiliated Hospital of Nantong University, Nantong 226001, Jiangsu Province, China

bepartment of Urology, The First Affiliated Hospital of Nanjing Medical University, Nanjing 210029, Jiangsu Province, China

'Department of Urology, The Sixth People's Hospital of Nantong, Nantong 226001, Jiangsu Province, China

${ }^{\mathrm{d}}$ These authors contributed equally to this work.

${ }^{e}$ Corresponding Author: Qianwei Xing, Department of Urology, Affiliated Hospital of Nantong University, Nantong 226001, Jiangsu Province, China. Email: xingqianwei@ntu.edu.cn; Bingye Zhu, Department of Urology, The Sixth People's Hospital of Nantong, Nantong 226001, Jiangsu Province, China. Email: zhubingye1995@163.com

doi: https://doi.org/10.14740/wjon1446
(TMB), immune microenvironment, immune cells, immune checkpoints and immune infiltration (all $\mathrm{P}<0.05$ ). Analysis of tumor immune dysfunction and exclusion (TIDE) and Imvigor210 datasets suggested that patients with low PPP1R18 expression are more likely to benefit from immunotherapy. Finally, we identified two potential mechanisms of a classical competing endogenous RNA (ceRNA) mechanism and an RNA-binding protein (RBP) involved mechanism of PPP1R18 in KIRC.

Conclusions: PPP1R18 played oncogenic and immunological roles of OS in KIRC, offering potential antigens for developing KIRC mRNA vaccines.

Keywords: PPP1R18; Kidney renal clear cell carcinoma; Overall survival; Immunity; Mechanism

\section{Introduction}

According to the most recent 2022 assessment of the American Cancer Society, the estimated number of new cases of kidney and renal pelvic cancer in men and women is 50,290 and 28,710, respectively. The estimated number of deaths in men and women is 8,960 and 4,960, separately [1]. As for kidney renal clear cell carcinoma (KIRC), it accounts for the highest proportion of renal cancer and it is often highly insidious with no obvious symptoms until the disease has progressed to an advanced stage [2]. In the era of cancer precision medicine, cancer personalized therapy today could be much easier to be facilitated with the knowledge of cancer-specific genomics [3, 4]. Moreover, inspired by the recent article (DOI: https://doi.org/10.1186/ s12943-021-01480-x) published in Molecular Cancer, Yu et al revealed the double mechanisms of hsa circ_0003258 in prostate cancer $(\mathrm{PCa})$ progression by a classical competing endogenous RNA (ceRNA) mechanism (hsa_circ_0003258/IGF2BP3/ HDAC4 axis) and an RNA-binding protein (RBP) involved mechanism (hsa circ 0003258/miR-653-5p/ARHGAP5 axis), indicating that hsa circ 0003258 could act as an attractive biomarker or therapeutic target for $\mathrm{PCa}$ [5]. Hence, in this article, we also mined The Cancer Genome Atlas (TCGA) database to provide novel biomarkers to predict KIRC patients' prognosis and to explore its potentially double mechanisms, offering potential antigens for developing KIRC mRNA vaccines. 
By means of TCGA data mining, protein phosphatase 1 regulatory subunit 18 (PPP1R18) was finally identified by us in KIRC, after a series of gene filtrations including difference analysis, survival analysis, univariate analysis, multivariate analysis, other datasets and polymerase chain reaction (PCR) validations, sequentially. To our knowledge, PPP1R18, also known as HKMT1098, KIAA1949, was reported to be a protein phosphatase 1 (PP1)-binding protein, or an actin-regulatory protein $[6,7]$. Matsubara et al revealed that PPP1R18 was able to regulate osteoclasts' bone resorption activity, maturation and actin ring formation [6]. Yasuda et al shed light on that PPP1R18 could suppress the transcriptional activity of NFATc1 to repress osteoclast differentiation at early stage [7]. As for cancer research, Ha et al identified eight differentially expressed genes above 16-fold changes including PPP1R18, that were significantly related to preoperative chemoradiation therapy responses in rectal cancer [8]. Wang et al obtained 11 methylation driver genes containing PPP1R18, to be significantly associated with smoking in tumors [9]. In addition, expression levels of PPP1R18 were also reported to be decreased in breast cancer cells, suggesting an anticancer effect [10]. However, currently little was known about the roles of PPP1R18 in KIRC. In the present study, we aimed to investigate the immunological and prognostic roles of PPP1R18 for overall survival (OS) in KIRC patients, as well as the prediction of its potential mechanisms, providing some references for future researches.

\section{Materials and Methods}

\section{Data acquisition and processing}

We obtained high-throughput sequencing data, survival data and corresponding clinical information from the TCGA database (http://cancergenome.nih.gov/) for patients with KIRC, including 539 tumor samples as well as 72 normal samples. The expression levels of PPP1R18 were then extracted using R software (https://www.r-project.org/) to combine with clinical information for subsequent analysis and samples lacking survival data were excluded. In addition, sequencing data from the GEO database (GSE6344; https://www.ncbi.nlm.nih. gov/geo/) and the International Cancer Genome Consortium (ICGC) dataset were processed separately and used as external datasets to validate PPP1R18 expression. Finally, both the $\mid \log _{2}$ fold change $\mid \geq 2$ and the adjusted P-value $<0.05$ were considered to be significant.

\section{The performance of PPP1R18 in pan-cancer analysis}

By applying a single gene tool on Sangerbox website (http:// sangerbox.com/), we obtained the performance of PPP1R18 in pan-cancer analysis. Based on previously published studies [11] and the Simple Nucleotide Variation dataset of level 4 for all TCGA samples processed by MuTect2 software [12], microsatellite instability (MSI) scores and tumor mutational burden (TMB) scores for each tumor were obtained [13], in- tegrating with gene expression data. The significance of differences was analyzed using unpaired Wilcoxon rank sum and signed rank tests, respectively. P-values less than 0.05 were considered significant for all above analyses. As for the immunological aspects, we also applied the same single gene tool on Sangerbox website (http://sangerbox.com/) to investigate the relationships between PPP1R18 and tumor immune infiltration, tumor microenvironment in KIRC and to reveal the associations between PPP1R18 and immune checkpoint molecules, immune cells pathway in pan-cancer analysis, with Pearson's method and the threshold was $\mathrm{P}<0.001$ [14-16].

\section{Univariate/multivariate Cox regression analyses and gene set enrichment analysis (GSEA) in KIRC}

Univariate and multivariate Cox regression analyses were implemented to figure out whether or not PPP1R18 expression could significantly relate to OS and serve as an independent prognostic factor in KIRC. As described in previously published studies [17-19], PPP1R18-related pathways in this study were also investigated by applying GSEA.

\section{The verification of PPP1R18 expression by quantitative real-time PCR (qRT-PCR)}

To further verify the expression of PPP1R18, normal renal tubular cell line HK-2 and tumor cell lines Caki-1, Caki-2, 786, $769, \mathrm{ACHN}$, and 10 pairs of tumor and adjacent non-tumor tissues collected from KIRC patients of the Affiliated Hospital of Nantong University were used for subsequent study. We used the TRIzol reagent (Invitrogen, Carlsbad, CA, USA) on kidney cancer cell lines and ground tissue to extract total RNA and Thermo Fisher's reverse transcription kit was used to obtain the corresponding cDNA based on the manufacturer's guidance. Then the qRT-PCR was implemented by applying QuantStudio5 Real-Time PCR System (Applied Biosystems, Foster City, CA, USA), using ChamQ SYBR qPCR Master Mix (Vazyme). Primers involved in this article were: PPP1R18 (F: 5'-AGGCCCATAAATGGAGACCTG-3'; R: 5'-TTTTGGAGCTACCCCTGGAAC-3') and $\beta$-actin (F: 5'-ATGACTTAGTTGCGTTACACC-3'; R: 5'-GACTTCCTGTAACAACGCATC-3'). Graphpad Prism 8 was used for data analysis and the creation of illustrations. Calculation approach of relative mRNA levels was $2^{-\Delta \Delta C t}$ method. This study was approved by the Institutional Research Ethics Committees of the Affiliated Hospital of Nantong University.

\section{Prediction of response to immunotherapy}

We used tumor immune dysfunction and exclusion (TIDE) database to predict the effect of immunotherapy by uploading the expression matrix to the website (http://tide.dfci.harvard.edu) for further calculation [20]. Imvigor210 data were also downloaded for predicting immunotherapy response [21]. Both were considered significant when $\mathrm{P}$ values less than 0.05 . 


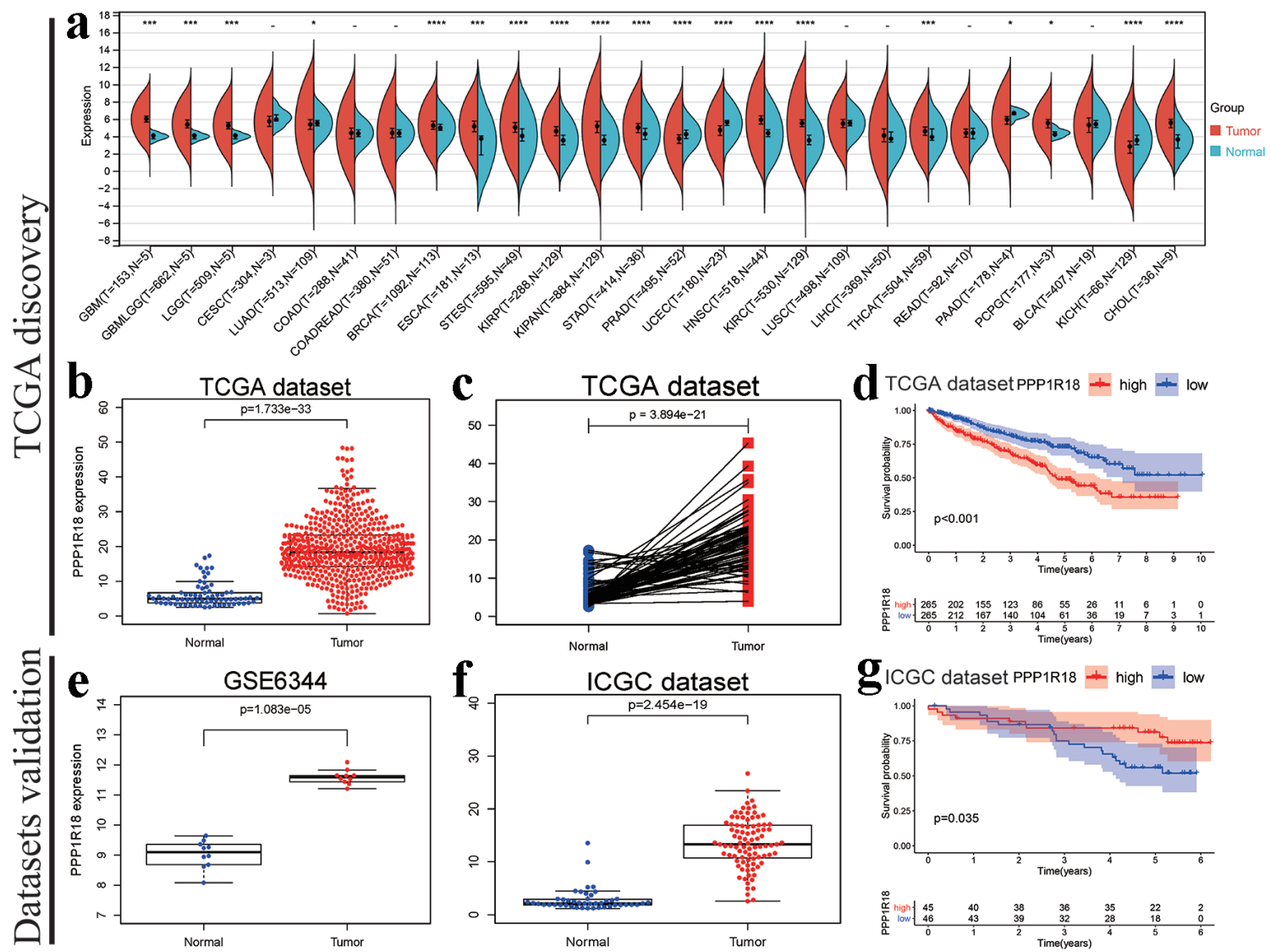

Figure 1. Prognostic roles of PPP1R18 involved in KIRC: (a) PPP1R18 mRNA expression levels in TCGA pan-cancer; (b) Boxplot of PPP1R18 mRNA expression levels in TCGA-KIRC dataset; (c) pairwise boxplot of PPP1R18 mRNA expression levels in TCGA-KIRC dataset; (d) K-M survival analysis of PPP1R18 in TCGA-KIRC dataset; (e) PPP1R18 mRNA expression levels in GSE6344; (f) PPP1R18 mRNA expression levels in ICGC dataset; (g) K-M survival analysis of PPP1R18 in ICGC dataset. *P < 0.05 ; ${ }^{* *} \mathrm{P}<0.01$; ${ }^{* *} \mathrm{P}<0.001$; ${ }^{* * *} \mathrm{P}<0.0001$. PPP1R18: protein phosphatase 1 regulatory subunit 18; KIRC: kidney renal clear cell carcinoma; TCGA: The Cancer Genome Atlas; ICGC: International Cancer Genome Consortium.

\section{Exploration of double mechanisms of PPP1R18}

As for the potential mechanisms of PPP1R18 involved in KIRC, we referred to previous published articles [22-24] and design two approaches to identify two potential mechanisms of a classical ceRNA mechanism (LncRNA/miRNA/ PPP1R18 mRNA networks) and an RBP involved mechanism (LncRNA/RBP/PPP1R18 mRNA networks) with the help of starBase v2.0 (http://starbase.sysu.edu.cn) database. With the successive threshold of pan-cancer $\geq 10$ cancer types, strict stringency $(\geq 5)$, hub LncRNAs in TCGA-KIRC and LncRNAs positively correlated with PPP1R18 in TCGAKIRC, the LncRNA/RBP/PPP1R18 mRNA networks were identified. As for PPP1R18-related ceRNA mechanism, we adjusted the criteria to programNum $\geq 2$, miRNAs negatively correlated with PPP1R18 in TCGA-KIRC, Hub miRNAs in TCGA-KIRC, Hub LncRNAs in TCGA-KIRC, miRNAs negatively correlated with LncRNAs in TCGA-KIRC, LncRNAs positively correlated with PPP1R18 in TCGA-KIRC, low stringency $(\geq 1)$. Finally, the LncRNA/miRNA/PPP1R18
mRNA networks were established. Both of them were visualized by Cytoscape 3.6.0.

\section{Results}

\section{The expression and prognosis of PPP1R18 in KIRC}

In this study, our results indicated that PPP1R18 had significant expression differences in various cancers in TCGA database, including KIRC (all $\mathrm{P}<0.05$, Fig. 1a-c). Moreover, based on the median expression, elevated expression of PPP1R18 was markedly associated with poorer OS than in its low expression group in TCGA-KIRC dataset $(\mathrm{P}<0.001$, Fig. 1d). We further utilized the GSE6344, ICGC datasets as external verifications to verify the expression and survival differences of PPP1R18 in KIRC and the results remained to be consistent (all $\mathrm{P}<0.05$, Fig. 1e-g).

By our own data validations, PPP1R18 was highly expressed in Caki-1, 769 renal cancer cell lines than in HK-2 
by $2^{-\Delta \Delta C t}$ methods (Fig. 2a). Furthermore, PPP1R18 also had higher expressions by $2^{-\Delta \Delta C t}$ methods in 10 pairs of KIRC tissues obtained from Affiliated Hospital of Nantong University (Fig. 2b). Further univariate and multivariate analyses indicated that PPP1R18 could be an independent biomarker of OS for KIRC (both $\mathrm{P}<0.05$, Table 1). All of above mentioned results showed the prognostic roles of PPP1R18 in KIRC.

\section{PPP1R18-related signaling pathways identified by GSEA}

As to single gene related pathways, our results indicated that PPP1R18 was significantly related to Chemokine, JAK STAT, MAPK, NOTCH, VEGF, B-cell receptor, T-cell receptor, Nodlike receptor, Toll-like receptor signaling pathways by means of GSEA with the threshold of |Normalized Enrichment Score $(\mathrm{NES}) \mid>1.5$ and nominal $\mathrm{P}<0.05$ (Table 2).

\section{Relationships between PPP1R18 and MSI, TMB in KIRC and its potential response to immunotherapies}

As for MSI and TMB, our results shed light on that PPP1R18 was remarkably linked to not only MSI ( $P=0.0049$, Fig. $2 \mathrm{c})$, but also TMB ( $\mathrm{P}=0.01$, Fig. $2 \mathrm{~d})$. To further predict the immune responses of PPP1R18 to immunotherapies, TIDE database and the IMvigor210 cohort were utilized, respectively. Based on above mentioned results, KIRC patients with low-PPP1R18 expression shall have a lower TIDE score, and be more sensitive to immunotherapies (both $\mathrm{P}<0.05$, Fig. 2e, f). Taken together, PPP1R18 could serve as a biomarker for immunotherapies and patients with low-PPP1R18 expression shall benefit most in KIRC.

\section{Associations between PPP1R18 and immunity}

To further explore the immunological roles of PPP1R18 involved in KIRC, we analyzed the relationships between PPP1R18 and immune infiltration levels. According to the threshold of correlation coefficient $>0.3$ and $\mathrm{P} \leq 0.0001$, our results presented that PPP1R18 was significantly correlated with immune infiltration levels of all six cells (Fig. 3). As for tumor microenvironment (TME), immune cells, immune checkpoint molecules, according to the same threshold, our results demonstrated that PPP1R18 was markedly related to TME of ESTIAMTE, immune, stromal scores in KIRC (Fig. 4a-c). As displayed in Figure $4 \mathrm{~d}$, e, PPP1R18 was also proved to be dramatically linked to immune checkpoint molecules like CD274 (PDL1), PDCD1 (PD1), CTLA4, BTLA, etc. (all P < 0.05) and immune cells like activated B cells, activated CD4 T cells, activated CD8 T cells, etc. (all $\mathrm{P}<0.05)$ in KIRC. All of these indicated that PPP1R18 was significantly correlated with immunity in KIRC.

\section{Construction of classical ceRNA and RBP-related net- works}

After studying potential mechanisms of PPP1R18 involved in
KIRC based on the whole workflow in Supplementary Material 1 (www.wjon.org), PPP1R18 positively correlated with LncRNAs in KIRC was defined as correlation coefficient $\geq 0.4$ and $\mathrm{P} \leq 0.001$. Hub LncRNAs in KIRC were defined as differently expressed LncRNAs associated with significant $\mathrm{OS}(\mathrm{P} \leq$ $0.05)$. Finally, the LncRNA/RBP/PPP1R18 mRNA networks in KIRC were displayed in Figure 5a. As for the classical ceRNA mechanism, according to the whole workflow in Supplementary Material 1 (www.wjon.org), negatively or positively correlated genes in KIRC were defined as correlation coefficient $\geq 0.2$ and $\mathrm{P} \leq 0.001$. Hub miRNAs or LncRNAs in KIRC were defined as differently expressed miRNAs or LncRNAs associated with significant $\mathrm{OS}(\mathrm{P} \leq 0.05)$. Eventually, the LncRNA/ miRNA/PPP1R18 mRNA networks in KIRC were presented in Figure 5b. All in all, Figure 5c summarized the diagram of PPP1R18 potentially involved mechanisms in KIRC.

\section{Discussion}

KIRC is the most common type of renal cell carcinoma, accounting for approximately $70-80 \%$ of cases, and it has the worst prognosis. Less than $15 \%$ of patients will present with the classic symptoms of abdominal pain, hematuria, and a mass [25-27]. The mortality rate of patients with KIRC has remained high due to lack of sensitivity to radiotherapy, and we have explored many areas such as molecularly targeted therapies [28, 29]. Given its high incidence and poor prognosis, there is a great need to find new molecular markers with diagnostic and prognostic significance and new prognostic signatures with excellent performance.

In this article, it was found that PPP1R18 is highly expressed in tumor tissues of KIRC patients, and significantly correlated to a poor prognosis of KIRC patients. Moreover, the ability of being an independent prognostic factor was confirmed after the univariate and multivariate Cox regression analyses. It is universally admitted that the evidence of differently expressed PPP1R18 being an important role was accumulating in various cancers. In earlier studies, it was demonstrated that there was significant variability of PPP1R18 in patients who responded to preoperative radiotherapy well for rectal cancer [8]. This study has guided treatment to some extent, and we can use PPP1R18 to carry out screening of radiotherapy-sensitive groups. Wang et al revealed that PPP1R18 was found to be a possible methylation driver gene associated with smoking-induced tumors on cancer patients with different smoking histories [9]. Thus, our study on the aspect of the relationship between PPP1R18 and survival of KIRC patients further complements the expression of this gene in numerous tumors, especially in KIRC.

There is no doubt that high expression of PPP1R18 is strongly associated with poor prognosis in KIRC patients. However, PPP1R1 8 involved pathways in KIRC remained unclear. Based on the different expression of PPP1R18, we performed GSEA enrichment analysis and results showed that it was enriched in Chemokine, JAK STAT, B-cell receptor and Tcell receptor signaling pathways. As for the Chemokine pathway, it has been demonstrated that it was an important pathway 


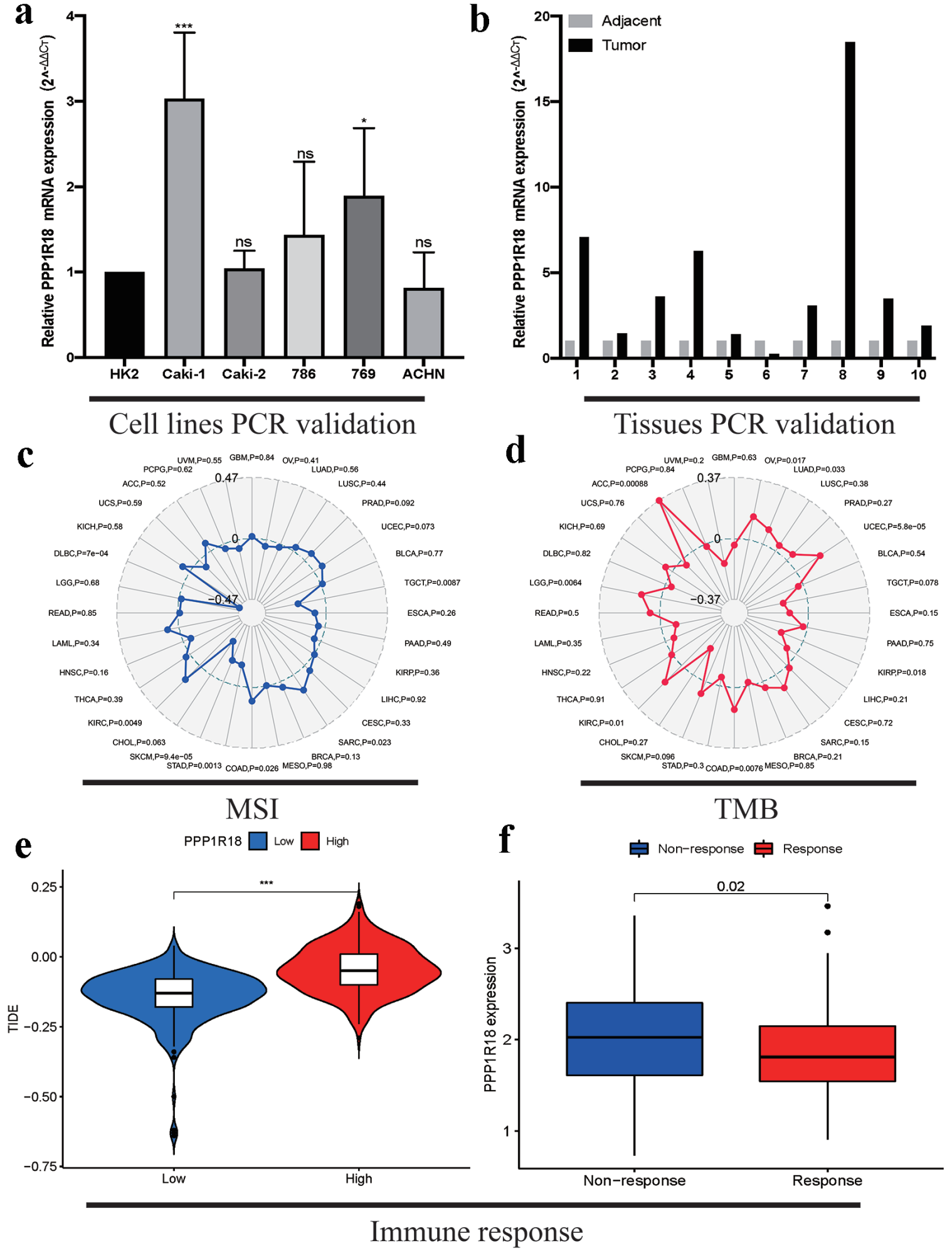

Figure 2. Verification of expression, MSI, TMB and immunotherapy response of PPP1R18 in KIRC: (a) PCR validations in renal cancer cell lines; (b) PCR validations in KIRC tissues; (c) associations between PPP1R18 expression and MSI; (d) associations between PPP1R18 expression and TMB; (e) prediction of immune response by TIDE database; (f) prediction of immune response by IMvigor210 cohort. ${ }^{*} \mathrm{P}<0.05$; ${ }^{* *} \mathrm{P}<0.001$. ns: no significance; TMB: tumor mutational burden; MSI: microsatellite instability; PPP1R18: protein phosphatase 1 regulatory subunit 18; KIRC: kidney renal clear cell carcinoma; TIDE: tumor immune dysfunction and exclusion; PCR: polymerase chain reaction. 
Table 1. Univariate and Multivariate Analyses of PPP1R18 and Several Clinical Factors for OS in TCGA-KIRC Dataset

\begin{tabular}{|c|c|c|c|c|c|c|c|c|}
\hline \multirow{2}{*}{$\begin{array}{l}\text { Clinical } \\
\text { factors }\end{array}$} & \multicolumn{4}{|c|}{ Univariate analysis } & \multicolumn{4}{|c|}{ Multivariate analysis } \\
\hline & HR & HR.95L & HR.95H & P-value & HR & HR.95L & HR.95H & P-value \\
\hline Age & 1.033274 & 1.019678 & 1.047052 & $1.28 \times 10^{-6}$ & 1.039339 & 1.023979 & 1.05493 & $3.79 \times 10^{-7}$ \\
\hline Race & 1.193075 & 0.71596 & 1.988138 & 0.498059 & 1.098543 & 0.640712 & 1.883524 & 0.73261 \\
\hline Grade & 1.966884 & 1.638836 & 2.360598 & $3.70 \times 10^{-13}$ & 1.367854 & 1.086933 & 1.721381 & 0.00757 \\
\hline M & 2.099647 & 1.660681 & 2.654644 & $5.70 \times 10^{-10}$ & 0.9157 & 0.484497 & 1.730671 & 0.786276 \\
\hline $\mathrm{N}$ & 0.862971 & 0.73887 & 1.007916 & 0.062826 & 0.847414 & 0.721356 & 0.995501 & 0.043922 \\
\hline PPP1R18 & 1.04568 & 1.028042 & 1.06362 & $2.66 \times 10^{-7}$ & 1.027619 & 1.008643 & 1.046953 & 0.004171 \\
\hline
\end{tabular}

PPP1R18: protein phosphatase 1 regulatory subunit 18; KIRC: kidney renal clear cell carcinoma; TCGA: The Cancer Genome Atlas; OS: overall survival.

related to KIRC by some bioinformatics analyses [30] and is associated with poor prognosis in immunophenotyping subtype $\mathrm{C} 1$ [31]. As to the JAK STAT pathway, the study of Zhang et al and Wang et al showed that both focal pyrotosis-related genes and ARNTL2 may affect KIRC through this pathway. And there are some links between these genes and the immune microenvironment $[32,33]$. Overall, there was a great need for relevant studies to analyze how this molecule was involved in the pathway and thus affected the progression of KIRC.

It is admitted that MSI occurs because DNA mismatch repair occurs in tumor tissues, while the outcome of TMB is highly correlated with the efficacy of PD1/PDL1 inhibitors. In our study, the expression of PPP1R18 correlated with both MSI and TMB. On one hand, the relationship between this gene and MSI predicts that PPP1R18 has the potential to become a new marker of KIRC, offering help in early detection of KIRC. On the other hand, the results of TMB analysis suggested that patients with low expression of PPP1R18 are more likely to benefit from treatment with PD1/PDL1 inhibitors, providing us with new evidence for the use of PD1/PDL1 inhibitors. As for the immunotherapy aspect of KIRC, the results of TIDE and Imvgiovr210 illustrate that patients with low expression of PPP1R18 are more likely to benefit from immunotherapy. All this part of the study provides a comprehensive prediction of the treatment of KIRC patients based on the expression of PPP1R18, providing new ideas for further drug trials.

Immunotherapy has been a popular means of tumor treatment, such as PD1/PDL1 inhibitors attacking tumors by activating the immune system. They can block the binding of PD1 and PDL1, increasing the proliferation of T cells, then it could enhance the recognition ability and killing function of $\mathrm{T}$ cells on tumor cells, and achieve anti-tumor effects [34, 35]. In our experimental results, the expression of PPP1R18 was very significantly associated with the immune microenvironment, immune cells, and immune checkpoints. This suggests that the expression of PPP1R18 in KIRC patients may affect the immune response and immune escape of tumors through these ways. Then the immune-related mechanisms and roles of PPP1R18 in KIRC patients are well worth exploring in subsequent experiments.

Exploration of the ceRNA mechanism of KIRC has been ongoing and there are many excellent studies. For example, Lv et al revealed that the circAGAP1/miR-15-5p/E2F3 mRNA

Table 2. GSEA Results of PPP1R18 Involved Pathways in TCGA-KIRC Dataset

\begin{tabular}{lllll}
\hline MSigDB collection & Gene set name & NES & NOM P-value & FDR Q-value \\
\hline c2.cp.kegg.v7.1.symbols.gmt & B_CELL_RECEPTOR_SIGNALING_PATHWAY & 2.225 & $<0.001$ & 0.001 \\
& CHEMOKINE_SIGNALING_PATHWAY & 2.517 & $<0.001$ & $<01$ \\
& JAK_STAT_SIGNALING_PATHWAY & 2.407 & $<0.001$ & $<0.001$ \\
& MAPK_SIGNALING_PATHWAY & 2.150 & $<0.001$ & 0.001 \\
& NOD_LIKE_RECEPTOR_SIGNALING_PATHWAY & 2.145 & 0.004 & 0.003 \\
& NOTCH_SIGNALING_PATHWAY & 2.191 & $<0.001$ & 0.002 \\
& T_CELL_RECEPTOR_SIGNALING_PATHWAY & 2.297 & $<0.001$ & $<0.001$ \\
& TOLL_LIKE_RECEPTOR_SIGNALING_PATHWAY & 2.210 & 0.006 & 0.002 \\
& VEGF_SIGNALING_PATHWAY & 2.032 & $<0.001$ & 0.006
\end{tabular}

GSEA: gene set enrichment analysis; PPP1R18: protein phosphatase 1 regulatory subunit 18; KIRC: kidney renal clear cell carcinoma; TCGA: The Cancer Genome Atlas. 

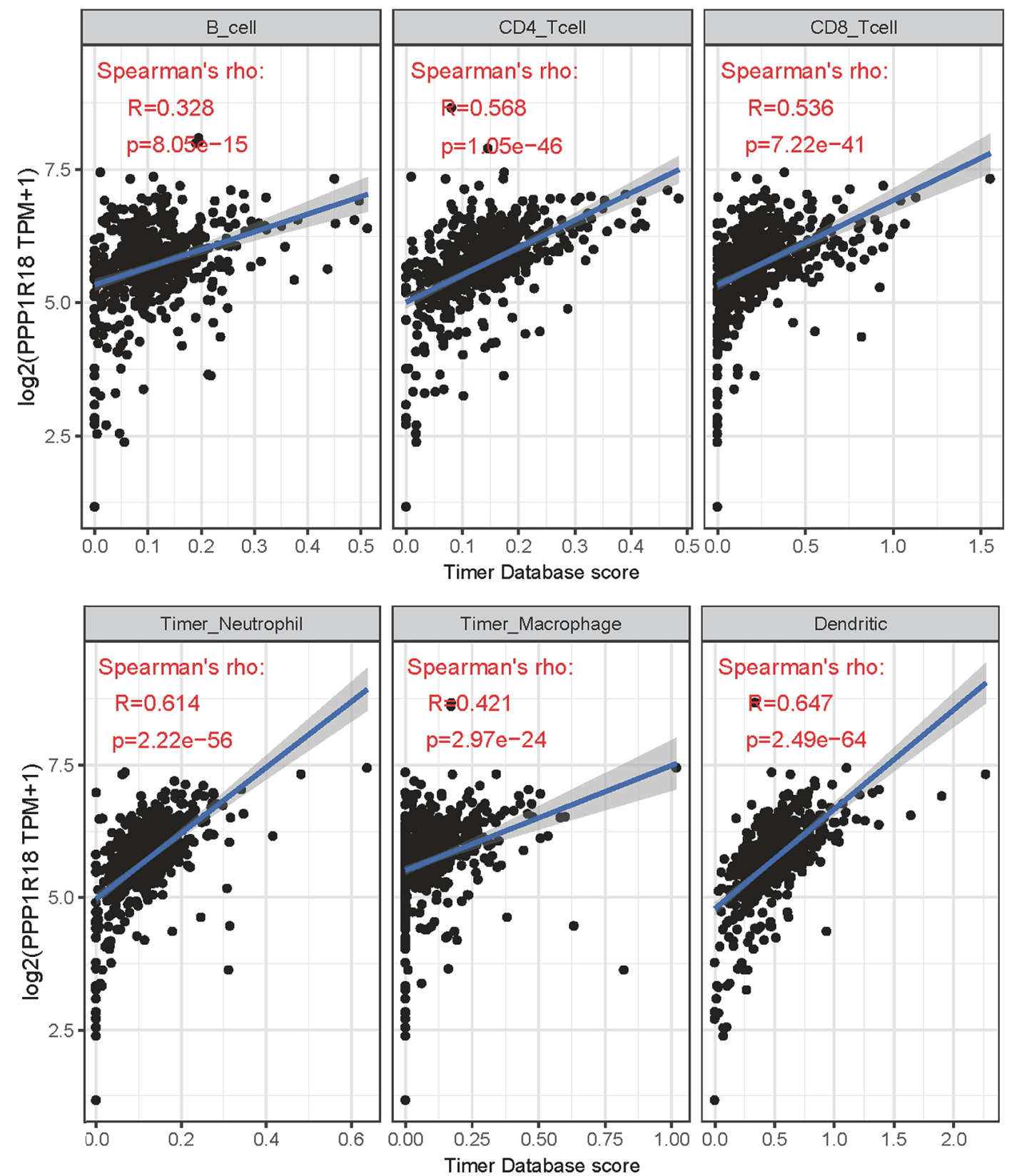

Figure 3. Relationships between PPP1R18 and immune infiltrations in KIRC. PPP1R18: protein phosphatase 1 regulatory subunit 18; KIRC: kidney renal clear cell carcinoma.

network was shown to be involved in tumor progression [36]. CircAGAP1 acts as a ceRNA to promote E2F3 expression via miR-15-5p and then exerts a pro-cancer function. Another lncPENG/miR-15b/PDZK1 mRNA network identified by Qi et al is involved in the regulation of tumor cell proliferation, in which LncPENG acts as a ceRNA to attenuate miR-15bdependent PDZK1 down-regulation and inhibit cell proliferation [37]. As for the RBP-related mechanisms, there are many outstanding studies involving KIRC too. A study revealed RBP SORBS2 inhibits KIRC transfer by enhancing MTUS1 mRNA stability [38]. The study by Zou et al showed that LINC00324 promotes gastric cancer cell proliferation with the help of binding to HuR and stabilizing FAM83B expression [39]. Besides, it was confirmed that EGFR-AS1 can enhance the malignant phenotype of RCC cells by enhancing HuR-mediated EGFR mRNA stability [22]. Encouraged by existing studies of ceRNA mechanisms and RBP-related mechanisms, we applied a scientific approach to make bold predictions and found that a classical ceRNA mechanism (LncRNA/miRNA/PPP1R18 mRNA networks) and a RBP-related mechanism (LncRNA/ RBP/PPP1R18 mRNA networks). They are new explorations of the mechanism of PPP1R18 mRNA in KIRC and need to be 

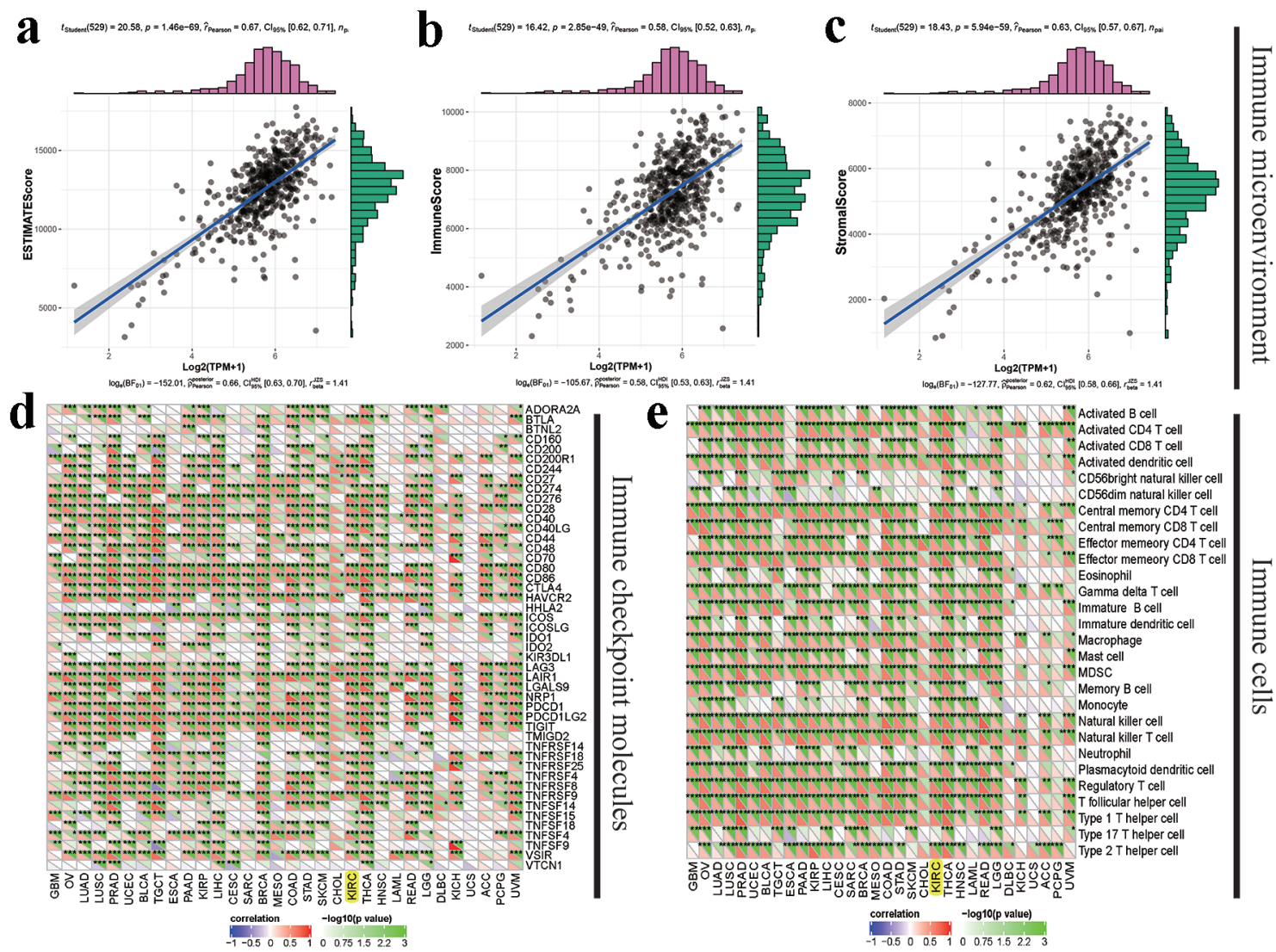

Figure 4. Immunological roles of PPP1R18 involved in KIRC: (a-c) relationships between PPP1R18 and tumor immune microenvironment in KIRC; (d) relationships between PPP1R18 and immune checkpoint molecules; (e) relationships between PPP1R18 and immune cells. ${ }^{*} \mathrm{P}<0.05 ;{ }^{* *} \mathrm{P}<0.01$; ${ }^{* *} \mathrm{P}<0.001$. PPP1R18: protein phosphatase 1 regulatory subunit 18 ; KIRC: kidney renal clear cell carcinoma.

a LncRNA/RBP/PPP1R18 mRNA axis

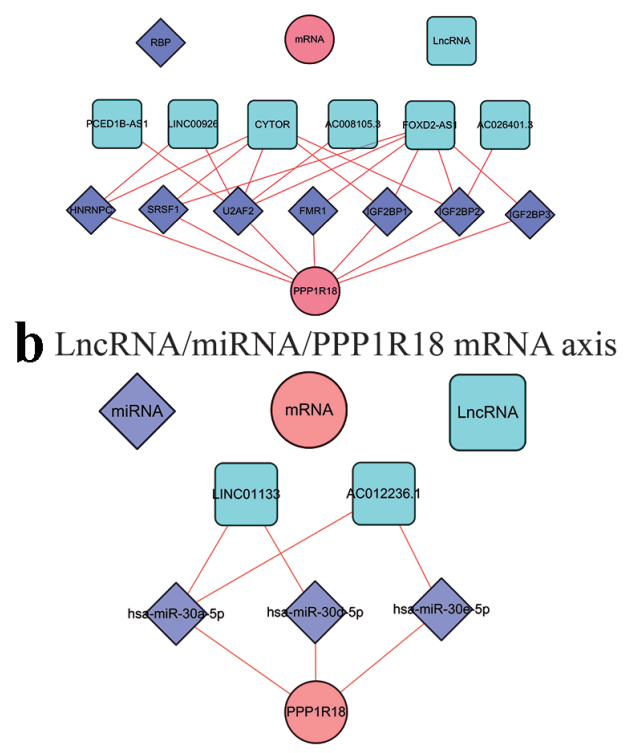

C Diagram of PPP1R18 involved mechanisms in KIRC

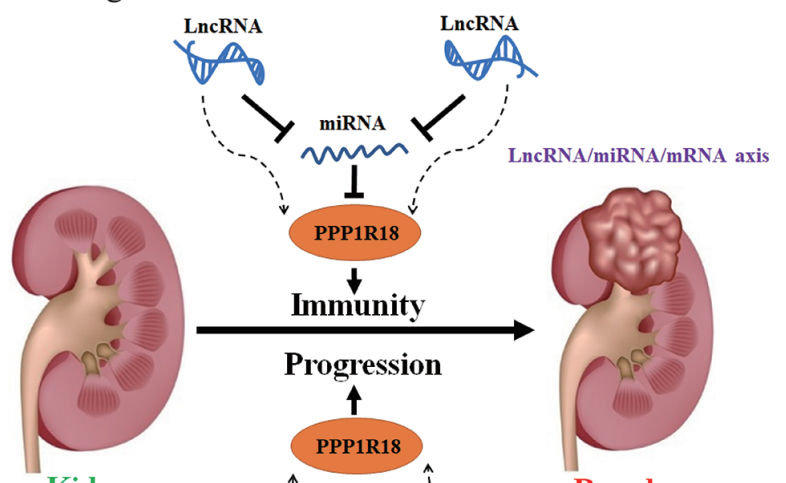

Kidney

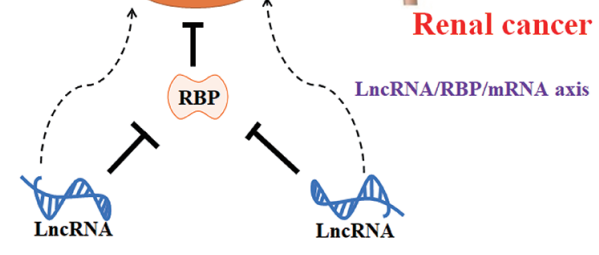

Figure 5. Potential mechanisms of PPP1R18 involved in KIRC: (a) LncRNA/RBP/PPP1R18 mRNA networks in KIRC; (b) LncRNA/miRNA/PPP1R18 mRNA networks in KIRC; (c) diagram of PPP1R18 involved mechanisms in KIRC. PPP1R18: protein phosphatase 1 regulatory subunit 18; KIRC: kidney renal clear cell carcinoma; RBP: RNA-binding protein. 
validated by follow-up studies.

As far as we knew, this was the first study to comprehensively explore the survival prognosis, tumor immune landscape, and immune responses of PPP1R18 in KIRC. We also utilized GSE6344, ICGC datasets and our own PCR results as external validations, making our results more stable and accurate. We finally identified two potential mechanisms of a classical ceRNA mechanism (LncRNA/miRNA/PPP1R18 mRNA networks) and an RBP involved mechanism (LncRNA/RBP/ PPP1R18 mRNA networks). However, there are some limitations in the design of this study. Firstly, the number of normal kidney samples in the TCGA and ICGC databases used was small at the stage of predicting the differences in PPP1R18 expression, and some bias may arise as a result. Then, we failed to validate the expression of PPP1R18 protein in renal cancer cell lines and renal tissue samples. Moreover, the clinical information from the database source was not diverse enough, without the history of smoking, alcohol consumption and radiotherapy, etc. Therefore, we need to collect more fresh specimens and corresponding clinical information to perform protein immunoblotting and immunohistochemical experiments for PPP1R18 and predicted related molecules to further validate the expression of PPP1R18 and mechanism-related molecules. In addition, experiments such as PPP1R18 knockdown were performed to validate the predicted classical ceRNA mechanism and RBP-related mechanism.

\section{Conclusion}

Taken together, PPP1R18 played an oncogenic role and could serve as an independent biomarker for OS in KIRC. Nine signaling pathways including Chemokine, JAK STAT, MAPK, NOTCH, VEGF, B-cell receptor, T-cell receptor, Nod-like receptor, and Toll-like receptor signaling pathways, were revealed to be involved with PPP1R18 in KIRC. Moreover, PPP1R18 was significantly correlated with immunity in KIRC and it could serve as a biomarker for immunotherapies with low-PPP1R18 expressed patients benefiting the most in KIRC, offering potential antigens for developing KIRC mRNA vaccines. We even identified two potential mechanisms of a classical ceRNA mechanism (LncRNA/miRNA/PPP1R18 mRNA networks) and an RBP involved mechanism (LncRNA/RBP/PPP1R18 mRNA networks) for its potential mechanisms. Further experiments were needed in order to test our findings in KIRC.

\section{Supplementary Material}

Suppl 1. The whole workflow of PPP1R18 involved mechanisms in KIRC: (A) The workflow of selecting LncRNA/RBP/ PPP1R18 mRNA axis in KIRC; (B) the workflow of selecting LncRNA/miRNA/PPP1R18 mRNA axis in KIRC.

\section{Acknowledgments}

None to declare.

\section{Financial Disclosure}

None to declare.

\section{Conflict of Interest}

None to declare.

\section{Informed Consent}

Not applicable.

\section{Author Contributions}

YW, SYL, and YHC: manuscript writing/editing; SYL, BYZ, and YHC: data analysis; QWX and YW: protocol/project development.

\section{Data Availability}

All data involved in this article were obtained from TCGAKIRC, GSE6344, and ICGC datasets.

\section{Abbreviations}

KIRC: kidney renal clear cell carcinoma; RBP: RNA-binding protein; TCGA: The Cancer Genome Atlas; GSEA: gene set enrichment analysis; OS: overall survival; TMB: tumor mutational burden; MSI: microsatellite instability; PPP1R18: protein phosphatase 1 regulatory subunit 18; PCR: polymerase chain reaction; PP1: protein phosphatase 1; NES: Normalized Enrichment Score; TIDE: tumor immune dysfunction and exclusion; TME: tumor microenvironment; ceRNA: competing endogenous RNA; ICGC: International Cancer Genome Consortium

\section{References}

1. Siegel RL, Miller KD, Fuchs HE, Jemal A. Cancer statistics, 2022. CA Cancer J Clin. 2022;72(1):7-33.

2. Luciani LG, Cestari R, Tallarigo C. Incidental renal cell carcinoma-age and stage characterization and clinical implications: study of 1092 patients (1982-1997). Urology. 2000;56(1):58-62.

3. Jahanafrooz Z, Mosafer J, Akbari M, Hashemzaei M, Mokhtarzadeh A, Baradaran B. Colon cancer therapy by focusing on colon cancer stem cells and their tumor microenvironment. J Cell Physiol. 2020;235(5):4153-4166.

4. Zardavas D, Pugliano L, Piccart M. Personalized therapy for breast cancer: a dream or a reality? Future Oncol. 2013;9(8):1105-1119. 
5. Yu YZ, Lv DJ, Wang C, Song XL, Xie T, Wang T, Li $\mathrm{ZM}$, et al. Hsa circ 0003258 promotes prostate cancer metastasis by complexing with IGF2BP3 and sponging miR-653-5p. Mol Cancer. 2022;21(1):12.

6. Matsubara T, Kokabu S, Nakatomi C, Kinbara M, Maeda T, Yoshizawa M, Yasuda H, et al. The actin-binding protein PPP1r18 regulates maturation, actin organization, and bone resorption activity of osteoclasts. Mol Cell Biol. 2018;38(4):e00425-1.

7. Yasuda K, Matsubara T, Shirakawa T, Kawamoto T, Kokabu S. Protein phosphatase 1 regulatory subunit 18 suppresses the transcriptional activity of NFATc1 via regulation of c-fos. Bone Rep. 2021;15:101114.

8. Ha YJ, Tak KH, Kim CW, Roh SA, Choi EK, Cho DH, Kim JH, et al. PSMB8 as a candidate marker of responsiveness to preoperative radiation therapy in rectal cancer patients. Int J Radiat Oncol Biol Phys. 2017;98(5):11641173.

9. Wang R, Li S, Wen W, Zhang J. Multi-Omics Analysis of the Effects of Smoking on Human Tumors. Front Mol Biosci. 2021;8:704910.

10. Su YA, Yang J, Tao L, Nguyen H, He P. Undetectable and decreased expression of KIAA1949 (Phostensin) encoded on chromosome $6 \mathrm{p} 21.33$ in human breast cancers revealed by transcriptome analysis. J Cancer. 2010;1:3850 .

11. Bonneville R, Krook MA, Kautto EA, Miya J, Wing MR, Chen HZ, Reeser JW, et al. Landscape of Microsatellite Instability Across 39 Cancer Types. JCO Precis Oncol. 2017;1:1-15.

12. Beroukhim R, Mermel CH, Porter D, Wei G, Raychaudhuri S, Donovan J, Barretina J, et al. The landscape of somatic copy-number alteration across human cancers. Nature. 2010;463(7283):899-905.

13. Attalla K, DiNatale RG, Rappold PM, Fong CJ, SanchezVega F, Silagy AW, Weng S, et al. Prevalence and landscape of actionable genomic alterations in renal cell carcinoma. Clin Cancer Res. 2021;27(20):5595-5606.

14. Peng D, Wei C, Zhang X, Li S, Liang H, Zheng X, Jiang $\mathrm{S}$, et al. Pan-cancer analysis combined with experiments predicts CTHRC1 as a therapeutic target for human cancers. Cancer Cell Int. 2021;21(1):566.

15. Liu J, Wang Y, Yin J, Yang Y, Geng R, Zhong Z, Ni $\mathrm{S}$, et al. Pan-cancer analysis revealed SRSF9 as a new biomarker for prognosis and immunotherapy. J Oncol. 2022;2022:3477148.

16. Ma L, Jin G, Yao K, Yang Y, Chang R, Wang W, Liu J, et al. Safety and Efficacy of Anti-PD-1/PD-L1 inhibitors compared with docetaxel for NSCLC: a systematic review and meta-analysis. Front Pharmacol. 2021;12:699892.

17. Hung JH, Yang TH, Hu Z, Weng Z, DeLisi C. Gene set enrichment analysis: performance evaluation and usage guidelines. Brief Bioinform. 2012;13(3):281-291.

18. Zhang SS, Zhu L, Peng Y, Zhang L, Chao FL, Jiang L, Xiao Q, et al. Long-term running exercise improves cognitive function and promotes microglial glucose metabolism and morphological plasticity in the hippocampus of APP/PS1 mice. J Neuroinflammation. 2022;19(1):34.

19. Zhu Z, Cao C, Zhang D, Zhang Z, Liu L, Wu D, Sun
J. UBE2T-mediated Akt ubiquitination and Akt/betacatenin activation promotes hepatocellular carcinoma development by increasing pyrimidine metabolism. Cell Death Dis. 2022;13(2):154.

20. Jiang P, Gu S, Pan D, Fu J, Sahu A, Hu X, Li Z, et al. Signatures of $T$ cell dysfunction and exclusion predict cancer immunotherapy response. Nat Med. 2018;24(10):15501558.

21. Hopkins AM, Kichenadasse G, Karapetis CS, Rowland A, Sorich MJ. Concomitant antibiotic use and survival in urothelial carcinoma treated with atezolizumab. Eur Urol. 2020;78(4):540-543.

22. Wang A, Bao Y, Wu Z, Zhao T, Wang D, Shi J, Liu B, et al. Long noncoding RNA EGFR-AS1 promotes cell growth and metastasis via affecting HuR mediated mRNA stability of EGFR in renal cancer. Cell Death Dis. 2019;10(3):154.

23. Wang Y, Tian Y, Liu S, Wang Z, Xing Q. Prognostic value and immunological role of AXL gene in clear cell renal cell carcinoma associated with identifying LncRNA/RBP/ AXL mRNA networks. Cancer Cell Int. 2021;21(1):625.

24. Wang W, Hu W, Wang Y, An Y, Song L, Shang P, Yue Z. Long non-coding RNA UCA1 promotes malignant phenotypes of renal cancer cells by modulating the miR-1825p/DLL4 axis as a ceRNA. Mol Cancer. 2020;19(1):18.

25. Inamura K. Renal Cell Tumors: Understanding Their Molecular Pathological Epidemiology and the 2016 WHO Classification. Int J Mol Sci. 2017;18(10):2195.

26. Jonasch E, Walker CL, Rathmell WK. Clear cell renal cell carcinoma ontogeny and mechanisms of lethality. Nat Rev Nephrol. 2021;17(4):245-261.

27. Ljungberg B, Bensalah K, Canfield S, Dabestani S, Hofmann F, Hora M, Kuczyk MA, et al. EAU guidelines on renal cell carcinoma: 2014 update. Eur Urol. 2015;67(5):913-924.

28. Holler N, Zaru R, Micheau O, Thome M, Attinger A, Valitutti S, Bodmer JL, et al. Fas triggers an alternative, caspase-8-independent cell death pathway using the kinase RIP as effector molecule. Nat Immunol. 2000;1(6):489495.

29. Scholtes MP, Alberts AR, Ifle IG, Verhagen P, van der Veldt AAM, Zuiverloon TCM. Biomarker-oriented therapy in bladder and renal cancer. Int J Mol Sci. 2021;22(6):2832.

30. Tian ZH, Yuan C, Yang K, Gao XL. Systematic identification of key genes and pathways in clear cell renal cell carcinoma on bioinformatics analysis. Ann Transl Med. 2019;7(5):89.

31. Bai D, Chen S, Feng H, Yin A, Lu J, Ma Y, Sugiyama H. Integrated analysis of immune-related gene subtype and immune index for immunotherapy in clear cell renal cell carcinoma. Pathol Res Pract. 2021;225:153557.

32. Du T, Gao J, Li P, Wang Y, Qi Q, Liu X, Li J, et al. Pyroptosis, metabolism, and tumor immune microenvironment. Clin Transl Med. 2021;11(8):e492.

33. Zhang H, Luo YB, Wu W, Zhang L, Wang Z, Dai Z, Feng $\mathrm{S}$, et al. The molecular feature of macrophages in tumor immune microenvironment of glioma patients. Comput Struct Biotechnol J. 2021;19:4603-4618.

34. Upadhaya S, Neftelinov ST, Hodge J, Campbell J. Chal- 
lenges and opportunities in the PD1/PDL1 inhibitor clinical trial landscape. Nat Rev Drug Discov. 2022.

35. Yamaguchi H, Hsu JM, Yang WH, Hung MC. Mechanisms regulating PD-L1 expression in cancers and associated opportunities for novel small-molecule therapeutics. Nat Rev Clin Oncol. 2022.

36. Lv Q, Wang G, Zhang Y, Shen A, Tang J, Sun Y, Ma C, et al. CircAGAP1 promotes tumor progression by sponging miR-15-5p in clear cell renal cell carcinoma. J Exp Clin Cancer Res. 2021;40(1):76.

37. Qi Y, Ma Y, Peng Z, Wang L, Li L, Tang Y, He J, et al. Long noncoding RNA PENG upregulates PDZK1 expression by sponging miR-15b to suppress clear cell renal cell carcinoma cell proliferation. Oncogene. 2020;39(22):44044420.

38. Lv Q, Dong F, Zhou Y, Cai Z, Wang G. RNA-binding protein SORBS2 suppresses clear cell renal cell carcinoma metastasis by enhancing MTUS1 mRNA stability. Cell Death Dis. 2020;11(12):1056.

39. Zou Z, Ma T, He X, Zhou J, Ma H, Xie M, Liu Y, et al. Long intergenic non-coding RNA 00324 promotes gastric cancer cell proliferation via binding with HuR and stabilizing FAM83B expression. Cell Death Dis. 2018;9(7):717. 